\title{
Clinical risk scores for the early prediction of severe outocomes in patients hospitalized for COVID-19: comment
}

\author{
Raffaella Rossio ${ }^{1} \cdot$ Mauro Tettamanti $^{2} \cdot$ Alessandro Nobili $^{2} \cdot$ Sergio Harari ${ }^{3} \cdot$ Pier Mannuccio Mannucci $^{1}{ }^{1}$.

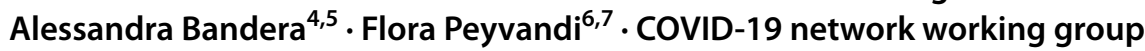

Received: 8 July 2021 / Accepted: 10 July 2021 / Published online: 3 August 2021

(c) Società Italiana di Medicina Interna (SIMI) 2021

\section{Dear Editor,}

On behalf of the Italian Society of Internal Medicine Ageno et al. [1] chose to use the database of a registry including 610 patients admitted with COVID-19 to five hospitals in Northern Italy in order to develop and validate a score that at the time of hospital admission would be able to predict a severe disease outcome, defined as the need of non-invasive ventilation, orotracheal intubation or death [1]. In the frame of their analysis meant to build the score and its accompanying validation the authors ultimately produced a score that, made of six clinical and laboratory variables, was able to identify patients at relatively low risk of a severe outcome who could be handled in the context of the low intensity of care offered by internal medicine wards [1]. During the selection

The members of COVID-19 network working group are mentioned in the Acknowledgement section.

Pier Mannuccio Mannucci

piermannuccio.mannucci@policlinico.mi.it

1 Angelo Bianchi Bonomi Hemophilia and Thrombosis Center, Fondazione IRCCS Ca' Granda Ospedale Maggiore Policlinico, Via Pace 9, 20122 Milano, Italy

2 Department of Neuroscience, Istituto Di Ricerche Farmacologiche Mario Negri IRCCS, Via Mario Negri 2, 20156 Milano, Italy

3 Division of Pulmonary and Critical Care Medicine, San Giuseppe Hospital MultiMedica IRCCS, Milan, Italy

4 Infectious Diseases Unit, Fondazione IRCCS Ca' Granda Ospedale Maggiore Policlinico, Milan, Italy

5 Department of Pathophysiology and Transplantation, University of Milan, Milano, Italy

6 Angelo Bianchi Bonomi Hemophilia and Thrombosis Center and Fondazione Luigi Villa, Fondazione IRCCS Ca' Granda Ospedale Maggiore Policlinico, Milan, Italy

7 Department of Pathophysiology and Transplantation, Università Degli Studi Di Milano, Milan, Italy and development of the best predictive score they chose to evaluate for inclusion data on demographic variables (age, sex, and time elapsed from disease onset), a few comorbidities (hypertension, diabetes, coronary artery disease, chronic obstructive pulmonary disease) but also an array of laboratory values obtained at admission, such as the white blood cells counts (WBC) (split by neutrophil and lymphocyte absolute counts), C-reactive protein (CRP), alanine amino transferase (ALT), aspartate amino transferase (AST), albumin, creatinine, D-dimer, and the neutrophil/lymphocyte ratio. Of these laboratory values CRP, AST, D-dimer, and the neutrophil/lymphocyte ratio were ultimately included in the actual score, with added clinical and demographic variables such as patient age and history of coronary artery disease [1].

In the context of our analysis of the database of another registry [2] implemented during the first wave of COVID-19 from February 2 to May 312020 in patients mainly admitted to two research hospitals acting as hubs in the metropolitan area of Milan (the capital city of Lombardy, the Italian region that was earlier and more severely hit by the SARS-CoV-2 pandemic) laboratory tests were carried out on admission in 1018 patients hospitalized with pneumonia and positive for SARS-CoV-2 RNA by RT PCR. After triage in the emergency room these patients were handled in the low- or intermediate care wards of pneumology, infectious disease or internal medicine. For our registry, we chose the laboratory tests with the main goal to ascertain the organs and body systems more compromised on admission in order to tailor on the basis of the corresponding results the type and intensity of medical support to organs and body systems other than the respiratory system. The latter, the principal one to be compromised by COVID-19, was supported by means of continuous positive airway pressure (CPAP), non-invasive ventilation (NIV) or high flow nasal cannula in patients admitted to pneumology wards, whereas those with relatively milder lung involvement were preferentially 
admitted to infectious disease and internal medicine wards, on oxygen supplementation provided mainly by means of nasal canula, ventimask and reservoir, more seldom by CPAP/NIV. The following tests were carried out: a complete blood count, including hemoglobin $(\mathrm{Hb})$, WBC (split by neutrophil and lymphocyte absolute counts) and platelets. Moreover, to assess the degree of systemic inflammation, serum CRP, ferritin and interleukin 6 (IL 6) were measured. Non-specific markers of tissue damage such as lactate dehydrogenase (LDH), ALT, and AST were also evaluated. The coagulation system was broadly explored by means of the prothrombin time international normalized ratio (INR) and D-dimer, the kidney function through the measurement of serum creatinine and the plasma electrolytes sodium and potassium. Finally, blood glucose was measured as an index of metabolic competence.

Having obtained at admission this battery of laboratory values we chose to describe and discuss herewith the results obtained in the patients who survived $(n=798)$ compared with those who died in hospital $(n=220)$ (Table 1). This comparison was carried out first by means of logistic regression analysis adjusted for sex and age but then also by multivariable logistic regression adjusted for all comorbidities as registered on admission. All blood tests were divided into four quartiles before being analyzed.

In the model corrected for age and sex, the blood components $\mathrm{Hb}$ and lymphocytes were significantly lower in non-survivors $(P$ value $<0.001)$ whereas the WBC and neutrophils were increased $(P<0.001)$, but the significance of the difference for $\mathrm{Hb}$ was lost in the fully adjusted multivariable analysis (Table 1). LDH was increased as nonorgan specific marker of tissue damage. Regarding the tests exploring blood coagulation, the prothrombin time INR was higher (and thus more abnormal) in non-survivors $(P=0.013$ after full adjustment). The mean values of D-dimer almost doubled in patients who ultimately died in hospital, a statistically significant difference from survivors maintained after full adjustment. Markers of systemic inflammation (CRP, ferritin and IL 6) were much higher in fatality cases, the statistical significance of the difference maintained after full adjustment $(P<0.02$ for both). Pertaining to markers of renal function, serum creatinine was higher even after full adjustment in patients who died $(P<0.001)$ but there was no difference for potassium and a small difference for sodium $(P=0.033)$. Blood glucose was higher in non-survivors.

These laboratory results obtained at hospital admission in patients with COVID-19 can be discussed with the results obtained by Ageno et al. [1] in the context of both their derivation and validation cohorts, even though they did not use laboratory markers to specifically evaluate the condition of body organs and systems, but to build up the prediction score. Moreover, they did not compare survivors with non-survivors, but patients with severe with those with non-severe COVID-19. Notwithstanding these differences their baseline results reported in their Table $1[1]$ are broadly similar to those obtained in the present registry.

Table 1 Main results of laboratory tests in survivors and non-survivors

\begin{tabular}{|c|c|c|c|c|c|c|}
\hline Laboratory test, median & Survivor & $(N=798)$ & Non-sur & vors $(N=220)$ & & $p$ value \\
\hline Haemoglobin (g/L) & $n=739$ & $13.0(11.7-14.2)$ & $n=201$ & $11.9(10.3-13.2)$ & $<0.001$ & 0.234 \\
\hline Lymphocytes $\left(\mathrm{mm}^{3}\right)$ & $n=682$ & 1055 (770-1450) & $n=186$ & $790(550-1030)$ & $<0.001$ & $<0.001$ \\
\hline Neutrophils $\left(\mathrm{mm}^{3}\right)$ & $n=684$ & $4475(3020-6520)$ & $n=187$ & $5960(4055-9810)$ & $<0.001$ & $<0.001$ \\
\hline $\mathrm{WBC}\left(\mathrm{mm}^{3}\right)$ & $n=737$ & $6180(4770-8330)$ & $n=202$ & $7475(5530-10,840)$ & $<0.001$ & $<0.001$ \\
\hline Platelets $\left(\mathrm{mm}^{3}\right)$ & $n=739$ & $230.000(170.000-291.000)$ & $n=201$ & $196.000(137.000-263.000)$ & 0.052 & 0.096 \\
\hline PT (INR) & $n=563$ & $1.1(1.0-1.2)$ & $n=150$ & $1.2(1.1-1.3)$ & 0.004 & 0.013 \\
\hline D-dimer $(\mathrm{mg} / \mathrm{L})$ & $n=434$ & $855(471-1656)$ & $n=119$ & $1493(953-3195)$ & $<0.001$ & 0.006 \\
\hline LDH (IU/L) & $n=423$ & $274(215-356)$ & $n=119$ & $405(293-512)$ & $<0.001$ & $<0.001$ \\
\hline AST (IU/L) & $n=377$ & $46(30-74)$ & $n=92$ & $55(38-92)$ & 0.194 & 0.118 \\
\hline ALT (IU/L) & $n=610$ & $37(22-59)$ & $n=180$ & $30(18-46)$ & 0.663 & 0.606 \\
\hline $\mathrm{C}$ reactive protein $(\mathrm{mg} / \mathrm{L})$ & $n=654$ & $6.1(2.7-11.3)$ & $n=183$ & $11.9(7.0-18.4)$ & $<0.001$ & $<0.001$ \\
\hline IL-6 (pg/mL) & $n=154$ & $25.2(9.8-61.6)$ & $n=35$ & $75.9(37.8-137.4)$ & 0.004 & 0.016 \\
\hline Ferritin $(\mathrm{ng} / \mathrm{ml})$ & $n=318$ & $754(348-1397)$ & $n=96$ & $843(450-1544)$ & 0.119 & 0.013 \\
\hline Creatinine (mg/dL) & $n=718$ & $0.9(0.7-1.1)$ & $n=202$ & $1.2(0.9-1.9)$ & $<0.001$ & $<0.001$ \\
\hline Sodium $(\mathrm{mmol} / \mathrm{L})$ & $n=710$ & $139(137-141)$ & $n=193$ & $140(136-144)$ & 0.004 & 0.033 \\
\hline Potassium (mmol/L) & $n=699$ & $4.1(3.8-4.4)$ & $n=187$ & $4.2(3.7-4.6)$ & 0.116 & 0.306 \\
\hline Glycemia (mg/dL) & $n=612$ & $105(92-125)$ & $n=174$ & $113(93-152)$ & $<0.001$ & 0.009 \\
\hline
\end{tabular}

Fully adjusted: age, body temperature, heart rate, respiratory rate, systolic BP, diastolic BP, number of comorbidities, number of drugs, dementia, chronic heart failure, cancer, diabetes, COPD, asthma, previous myocardial infarction, previous stroke/TIA, atrial fibrillation, malnutrition 
Our patients who died had higher WBC values, with a very high neutrophil to lymphocyte ratio owing to a decrease in lymphocytes. Inflammation markers such as CRP but also ferritin and IL6 were increased in non-survivors and this was also the case for creatinine, D-dimer and the PT INR, indicating that beside a marked inflammatory state COVID19 is often associated with an impairment of renal function and activation of the coagulation system. The laboratory results obtained in the context of the present registry can also be compared with those obtained in China at the time of the first 2020 wave of COVID-19, because Zhou et al. [3] compared like us 137 hospital survivors with 54 nonsurvivors. Like us they found that the lymphocyte count was significantly lower in non-survivors and that serum levels of a non-specific marker of tissue damage, such as LDH, were elevated.

Limitations of this analysis are that the laboratory markers were evaluated only at the time of hospital admission with no follow-up except for information on survival in hospital. However, a strength is the large sample of survivors and non-survivors. From these data obtained at the time of the first wave of COVID-19 in the most heavily and earlier hit region of Italy, we learnt that COVID-19 is a multiorgan disease, and that kidney function, glucose metabolism and the coagulation system must be closely monitored in patients presenting on admission with laboratory markers of organ involvement.

Acknowledgements Co-authors of the "COVID-19 network" working group are: Fondazione IRCCS Ca' Granda Ospedale Maggiore Policlinico. Scientific Direction: Silvano Bosari, Luigia Scudeller, Giuliana Fusetti, Laura Rusconi, Silvia Dell'Orto; Department of Transfusion Medicine and Hematology (Biobank): Daniele Prati, Luca Valenti, Silvia Giovannelli, Maria Manunta, Giuseppe Lamorte, Francesca Ferarri; Infectious Diseases Unit: Andrea Gori, Alessandra Bandera, Antonio Muscatello, Davide Mangioni, Laura Alagna, Giorgio Bozzi, Andrea Lombardi, Riccardo Ungaro, Giuseppe Ancona, Gianluca Zuglian, Matteo Bolis, Nathalie Iannotti, Serena Ludovisi, Agnese Comelli, Giulia Renisi, Simona Biscarini, Valeria Castelli, Emanuele Palomba, Marco Fava, Valeria Fortina, Carlo Alberto Peri, Paola Saltini, Giulia Viero, Teresa Itri, Valentina Ferroni,Valeria Pastore,Roberta Massafra,Arianna Liparoti,Toussaint Muheberimana, Alessandro Giommi, Rosaria Bianco, Rafaela Montalvao De Azevedo, Grazia Eliana Chitani; Angelo Bianchi Bonomi Hemophilia and Thrombosis Center and Fondazione Luigi Villa: Flora Peyvandi, Roberta Gualtierotti, Barbara Ferrari, Raffaella Rossio, Nadia Boasi, Erica Pagliaro, Costanza Massimo, Michele De Caro, Andrea Giachi; UOC Internal Medicine, Immunology and Allergology: Nicola Montano, Barbara Vigone, Chiara Bellocchi, Angelica Carandina, Elisa Fiorelli, Valerie Melli, Eleonora Tobaldini; Respiratory Unit and Cystic Fibrosis Adult Center: Francesco Blasi, Stefano Aliberti, Maura Spotti,Leonardo Terranova, Sofia Misuraca, Alice D’Adda, Silvia Della Fiore, Marta Di Pasquale, Marco Mantero, Martina Contarini, Margherita Ori, Letizia Morlacchi, Valeria Rossetti, Andrea Gramegna, Maria Pappalettera, Mirta Cavallini, Agata Buscemi; Cardiology Unit: Marco Vicenzi, Irena Rota, Giorgio Costantino, Monica Solbiati, Ludovico Furlan, Marta Mancarella, Giulia Colombo, Giorgio Colombo, Alice Fanin, Mariele Passarella; Acute
Internal Medicine: Valter Monzani, Ciro Canetta, Angelo Rovellini, Laura Barbetta, Filippo Billi, Christian Folli, Silvia Accordino; Rare Diseases Center: Diletta Maira, Cinzia Maria Hu, Irene Motta, Natalia Scaramellini; General Medicine and Metabolic Diseases: Anna Ludovica Fracanzani, Rosa Lombardi, Annalisa Cespiati ; Geriatric Unit: Matteo Cesari,Tiziano Lucchi,Marco Proietti, Laura Calcaterra, Clara Mandelli, Carlotta Coppola, Arturo Cerizza. Intensive Care Unit: Antonio Maria Pesenti, Giacomo Grasselli, Alessandro Galazzi. Istituto di Ricerche Farmacologiche Mario Negri IRCCS: Alessandro Nobili, Mauro Tettamanti, Igor Monti, Alessia Antonella Galbussera. Policlinico G.B. Rossi, Verona: UOC di Medicina d'Urgenza: Ernesto Crisafulli, Domenico Girelli, Alessio Maroccia, Daniele Gabbiani, Fabiana Busti, Alice Vianello, Marta Biondan, Filippo Sartori. Ospedale San Gerardo, ASST Monza: UOC Pneumologia: Paola Faverio, Alberto Pesci, Stefano Zucchetti. Malattie Infettive: Paolo Bonfanti, Marianna Rossi, Ilaria Beretta, Anna Spolti. San Giuseppe Hospital MultiMedica IRCCS and community health, Università degli Studi di Milano: UOC Pneumologia: Sergio Harari. Unità di Pneumologia e terapia Semi-intensiva respiratoria, Servizio di Fisiopatologia Respiratoria ed Emodinamica Polmonare: Davide Elia. Unità di Pneumologia e terapia Semi-intensiva respiratoria, Servizio di Fisiopatologia Respiratoria ed Emodinamica Polmonare: Roberto Cassandro, Antonella Caminati. Ospedale Clinicizzato "SS. Annunziata": Clinica Medica: Francesco Cipollone, Maria Teresa Guagnano, Damiano D'Ardes, Ilaria Rossi, Francesca Vezzani. ICS Maugeri Tradate, Universita' Insubria: Pneumologia Riabilitativa: Antonio Spanevello, Francesca Cherubino, Dina Visca. Azienda Ospedaliera Universitaria di Ferrara e Dipartimento di Medicina Traslazionale Università di Ferrara: UO Pneumologia: Marco Contoli, Alberto Papi, Luca Morandi, Nicholas Battistini. Clinica Polispecialistica San Carlo: UO Medicina Interna: Guido Luigi Moreo, Pasqualina Iannuzzi. UO Oncologia: Daniele Fumagalli. UO Chirurgia Generale: Sara Leone.

Silvano Bosari, Luigia Scudeller, Giuliana Fusetti, Laura Rusconi, Silvia Dell'Orto, Daniele Prati, Luca Valenti, Silvia Giovannelli, Maria Manunta, Giuseppe Lamorte, Francesca Ferarri, Andrea Gori, Alessandra Bandera, Antonio Muscatello, Davide Mangioni, Laura Alagna, Giorgio Bozzi, Andrea Lombardi, Riccardo Ungaro, Giuseppe Ancona, Gianluca Zuglian, Matteo Bolis, Nathalie Iannotti, Serena Ludovisi, Agnese Comelli, Giulia Renisi, Simona Biscarini, Valeria Castelli, Emanuele Palomba, Marco Fava, Valeria Fortina, CarloAlberto Peri, Paola Saltini, Giulia Viero, Teresa Itri, Valentina Ferroni,Valeria Pastore,Roberta Massafra,Arianna Liparoti,Toussaint Muheberimana, Alessandro Giommi, Rosaria Bianco, Rafaela Montalvao De Azevedo, GraziaEliana Chitani, Flora Peyvandi, Roberta Gualtierotti, Barbara Ferrari, Raffaella Rossio, Nadia Boasi, Erica Pagliaro, Costanza Massimo, Michele De Caro, Andrea Giachi, Nicola Montano, Barbara Vigone, Chiara Bellocchi, Angelica Carandina, Elisa Fiorelli, Valerie Melli, Eleonora Tobaldini, Francesco Blasi, Stefano Aliberti, Maura Spotti,Leonardo Terranova, Sofia Misuraca, Alice D’Adda, SilviaDella Fiore, Marta Di Pasquale, Marco Mantero, Martina Contarini, Margherita Ori, Letizia Morlacchi, Valeria Rossetti, Andrea Gramegna, Maria Pappalettera, Mirta Cavallini, Agata Buscemi, Marco Vicenzi, Irena Rota, Giorgio Costantino, Monica Solbiati, Ludovico Furlan, Marta Mancarella, Giulia Colombo, Giorgio Colombo, Alice Fanin, Mariele Passarella, Valter Monzani, Ciro Canetta, Angelo Rovellini, Laura Barbetta, Filippo Billi, Christian Folli, Silvia Accordino, Diletta Maira, CinziaMaria Hu, Irene Motta, Natalia Scaramellini, AnnaLudovica Fracanzani, Rosa Lombardi, Annalisa Cespiati, Matteo Cesari,Tiziano Lucchi,Marco Proietti, Laura Calcaterra, Clara Mandelli, Carlotta Coppola, Arturo Cerizza AntonioMaria Pesenti, Giacomo Grasselli, Alessandro Galazzi, Alessandro Nobili, Mauro Tettamanti, Igor Monti, AlessiaAntonella Galbussera, Ernesto Crisafulli, Domenico Girelli, Alessio Maroccia, Daniele Gabbiani, Fabiana Busti, Alice Vianello, Marta Biondan, Filippo Sartori, Paola Faverio, Alberto Pesci, Stefano Zucchetti, Paolo Bonfanti, Marianna Rossi, Ilaria Beretta, Anna Spolti, Sergio Harari, Davide Elia, Roberto Cassandro, Antonella Caminati, 
Francesco Cipollone, MariaTeresa Guagnano, Damiano D’Ardes, Ilaria Rossi, Francesca Vezzani, Antonio Spanevello, Francesca Cherubino, Dina Visca, Marco Contoli, Alberto Papi, Luca Morandi, Nicholas Battistini, GuidoLuigi Moreo, Pasqualina Iannuzzi, Daniele Fumagalli, Sara Leone.

Funding The authors received no financial support for this study.

\section{Declarations}

Conflict of interest The authors have no conflict of interest.

Human and animal rights statement This article does not contain any studies with human participants or animals performed by any of the authors.

Informed consent None.

\section{References}

1. Ageno W, Cogliati C, Perego M et al (2021) Clinical risk scores for the early prediction of severe outcomes in patients hospitalized for COVID-19. Intern Emerg Med 16:989-996

2. Bandera A, Nobili A, Tettamanti M, et al (2021) Clinical factors associated with death in 3044 COVID-19 patients managed in Internal Medicine wards in Italy: comment. Intern Emerg Med. https://doi.org/10.1007/s11739-021-02797-7

3. Zhou F, Yu T, Du R et al (2020) Clinical course and risk factors for mortality of adult inpatients with COVID-19 in Wuhan, China: a retrospective cohort study. Lancet 395:1054-1062

Publisher's Note Springer Nature remains neutral with regard to jurisdictional claims in published maps and institutional affiliations. 